\title{
A Survey of Mathematical Models of Dengue Fever
}

\author{
lurii Bakach ${ }^{1}$ and James Braselton ${ }^{2 *}$
}

${ }^{1}$ Department of Mathematics, 14 MacLean Hall, The University of lowa, lowa City, IA-52242-1419, USA

${ }^{2}$ Department of Mathematical Sciences, Southern University, Statesboro, Georgia-30460-8093, USA

\begin{abstract}
In this paper, we compare and contrast five models of dengue fever, a serious illness that affects tropical and subtropical areas around the world. We evaluate each model using different scenarios and identify the strengths and weakness of each of the models. The goal of our analysis is to indicate the strengths and weaknesses of current mathematical models of dengue fever that should assist future researchers in forming models that accurately measure the variables they are studying that affect the spread and progression of the disease.
\end{abstract}

Keywords: Dynamical system; SIR model; Dengue fever

\section{Introduction}

\section{Dengue fever}

Dengue is a mosquito-borne viral infection that is usually found in tropical and subtropical regions around the world. Warmer weather and rain bring excellent breeding grounds for mosquitos that are carriers and transmit the disease. As human population sizes increase in areas with these climates, there is an increase of the number of human carriers of the disease who when bitten by a mosquito can then infect the mosquito with a version of the virus that enables the mosquito to transmit the virus in subsequent bites to humans. In recent years, transmission has increased predominantly in urban and semi-urban areas and has become a major public health concern [1].

There are four distinct, but closely related, serotypes of the virus that cause dengue (DEN-1, DEN-2, DEN-3 and DEN-4). Recovery from infection by one provides lifelong immunity against that particular serotype. However, cross-immunity to the other serotypes after recovery is only partial and temporary. Subsequent infections by other serotypes increase the risk of developing severe dengue.

Accordingly to the World Health Organization (WHO) [1], over 2.5 billion people are now at risk for dengue. Currently, the WHO estimates that there may be 50-100 million dengue infections worldwide.

Not only is the number of cases increasing as the disease spreads to new areas, but explosive outbreaks are also occurring. The threat of a possible outbreak of dengue fever now exists in Europe and local transmission of dengue was reported for the first time in France and Croatia in 2010 and imported cases were detected in 10 other countries in Europe apart from mainland Portugal. In 2013, cases have occurred in Florida (United States of America) and Yunnan (province of China) [1].

Mathematical modelling is a powerful tool to test and compare different intervention strategies that might be useful in controlling or eliminating dengue, which is especially important in our world of limited resources. The various mathematical models help us conceptualize the transmission dynamics in a quantitative way as well as allow us to test different hypotheses to understand their importance.

In this paper we compare and contrast five different models of dengue fever and identify their best features along with their performance for various scenarios.

\section{The Basic $S-I-R$ Model and Concepts}

The $\mathrm{S}-\mathrm{I}-\mathrm{R}$ model is a basic model in which a constant population is divided into three compartments of people depending on their infection status: susceptible $S$, infected $I$ and recovered $R$.

This is usually called the $S-I-R$ model. The three compartments $S, I$, and $R$, are explained as follows:

- $S$ is used to represent the number of individuals who are susceptible to the disease at time $t$

- I denotes the number of individuals who have been infected with the disease and are capable of spreading the disease to those in the susceptible category

- $R$ represents the number of individuals who have been infected and recovered from the disease. Those in this category are immune to infection and they would not transmit the infection to others.

- Assumptions: Each compartment is assumed to be homogeneous. In other words, people in each compartment are randomly mixing with each other. This is similar to the mass action model in chemistry. The per capita rate of infection and the per capita rate of recovery are assumed to be independent of the length of time the person has spent in each compartment. They are assumed to follow an exponential distribution [2].

Consequently, the basic $S-I-R$ model can be formulated as follows:

$$
\begin{aligned}
& \frac{d S}{d t}=-\lambda S, \quad \frac{d I}{d t}=\lambda S-\gamma I, \\
& \frac{d R}{d t}=\gamma I, \quad S+I+R=N
\end{aligned}
$$

where $\lambda$ is the force of infection and $\gamma$ is the mean recovery rate.

*Corresponding author: James Braselton, Department of Mathematical Sciences, Southern University, Statesboro, Georgia 30460-8093, United States, Tel: +1912478-1966; E-mail: jbraselton@georgiasouthern.edu

Received July 27, 2015; Accepted August 06, 2015; Published August 08, 2015

Citation: Bakach I, Braselton J (2015) A Survey of Mathematical Models of Dengue Fever. J Comput Sci Syst Biol 8: 255-267. doi:10.4172/jcsb.1000198

Copyright: $\odot 2015$ Bakach I, et al. This is an open-access article distributed under the terms of the Creative Commons Attribution License, which permits unrestricted use, distribution, and reproduction in any medium, provided the original author and source are credited. 
$S-I-R$ models can have two separate formulations, depending on the basic assumptions regarding the force of infection: densitydependent and frequency dependent models.

- The density-dependent model: The density-dependent model assumes that members of a population existing in a fixed area all come in contact with one another no matter how many individuals are present in the population. Therefore, the force of infection is defined as $\lambda=\beta I$ where $\beta$ denotes the transmission coefficient (which is the product of the number of contact per susceptible person per unit time and the probability of a successful transmission of the infection given the contact). Assuming that $\beta$ is a constant, the frequency of infection depends on the number of infected persons in the population.

The frequency-dependent model: It has been shown that for most human infections, the number of people each person is in contact per day is fairly constant across the world, regardless of the population density of the place. That is why an alternative, known as the "frequency-dependent," formulation of the $S-I-R$ model is often used to model the transmission of human diseases, where the force of infection is defined as $\lambda=\beta\left(\frac{I}{N}\right)$. The term $\frac{I}{N}$ is the probability that any random contact that a susceptible person makes will be with someone infectious, which is equivalent to the proportion of the total population that is infectious.

It is important to clarify that all models considered here are frequency-dependent models.

\section{Derouich model of dengue fever}

We first study the model of dengue fever developed by Derouich et al. [3]. Their model is based on the compartmental diagram shown in Figure 1 . The host population, $N_{h}$, consists of susceptibles, $S_{h}$, infectives, $I_{h}$, and removed, $R_{h}$. The corresponding vector population, $N_{v}$, consists of susceptibles, $S_{v}$ and infectives $I_{v}$. Mosquitos are a reservoir host for the four viruses that cause dengue fever: they are carriers of the virus but not negatively affected by it. Hence, there is not a "removed vector population" to consider.

For the human population, the model developed by Derouich et al. [3], takes the form

$$
\begin{aligned}
& \frac{d S_{h}}{d t}=\mu_{h} N_{h}-\left(\mu_{h}+p+C_{v h} I_{v} / N_{h}\right) S_{h} \\
& \frac{d I_{h}}{d t}=\left(C_{v h} I_{v} / N_{h}\right) S_{h}-\left(\mu_{h}+\gamma_{h}\right) I_{h} \\
& \frac{d R_{h}}{d t}=\left(p S_{h}+\gamma\right) h I_{h}-\mu_{h} R_{h}
\end{aligned}
$$

The parameter values are described in Table 1 . One of the key features of the model is the fraction, $p$, which represents a (random) fraction of the human population that can be permanently immunized against the four serotypes that cause dengue fever.

For the vector population,

$$
\begin{aligned}
& \frac{d S_{v}}{d t}=\mu_{v} N_{v}-\left(\mu_{v}+C_{l w} I_{h} / N_{h}\right) S_{v} \\
& \frac{d I_{v}}{d t}=\left(C_{h v} I_{h} / N_{h}\right) S_{v}-\mu_{v} I_{v}
\end{aligned}
$$

Because $S_{h}+I_{h}+R_{h}=N_{h}$ and $S_{v}+I_{v}=N_{v}, R_{h}=N_{h}-S_{h}-I_{h}$ and $S_{v}=N_{v}-I_{v}$ equations (1) and (2) can be combined into the single system

$$
\begin{aligned}
& \frac{d S_{h}}{d t}=\mu_{h} N_{h}-\left(\mu_{h}+p+C_{v h} I_{v} / N_{h}\right) S_{h} \\
& \frac{d I_{h}}{d t}=\left(C_{v h} I_{v} / N_{h}\right) S_{h}-\left(\mu_{h}+\gamma_{h}\right) I_{h} \\
& \frac{d I_{v}}{d t}=\left(C_{h v} I_{h} / N_{h}\right)\left(N_{v}-I_{v}\right)-\mu_{v} I_{v}
\end{aligned}
$$

The main result of Derouich et al. [3] is that system (3) has two equilibrium points, $\mathrm{E}_{1}=\left(\mathrm{N}_{h}\left(1+\mathrm{p} / \mu_{h}\right), 0,0\right)$ and $\mathrm{E}_{2}=\left(\mathrm{S}_{h}{ }^{*}, \mathrm{I}_{h}{ }^{*}, \mathrm{I}_{v}{ }^{*}\right)$ where

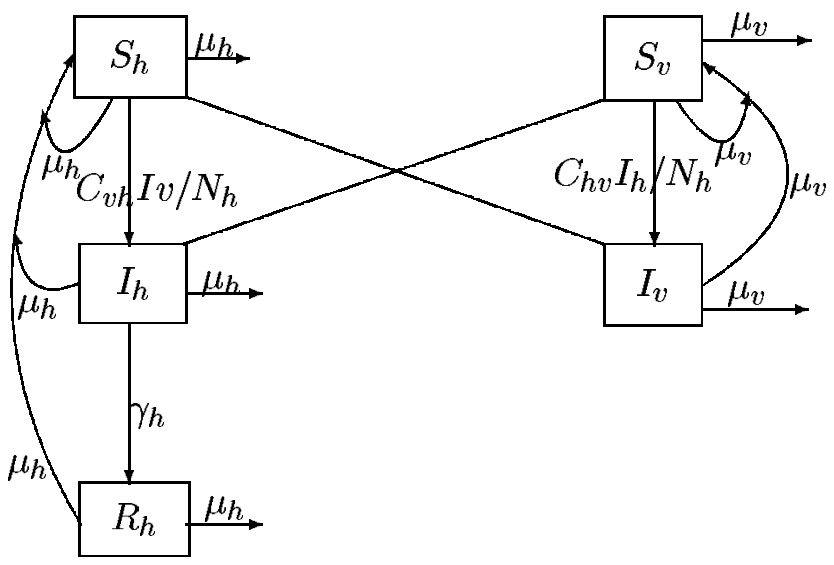

Figure 1: The compartmental diagram used by Derouich et al. [3] in the formulation of their model of Dengue fever.

\begin{tabular}{|l}
\hline Parameter \\
\hline Transmission probability of vector to human \\
Transmission probability of human to vector \\
Bites per susceptible mosquito per day \\
Bites per infectious mosquito per day \\
Effective contact rate: human to vector \\
Effective contact rate: vector to human \\
Human life span \\
Vector life span \\
Host infection duration
\end{tabular}

Table 1: Parameter values used following the same choices as in Derouich et al. [3]. 


$$
\begin{gathered}
S_{h}^{*}=\frac{N_{h}(\beta+M)}{\left(1+p / \mu_{h}\right) \beta+M R^{\prime}} \\
I_{h}^{*}=\frac{N_{h}\left(R-1-p / \mu_{h}\right)}{\left(1+p / \mu_{h}\right) \beta+M R^{\prime}} \\
I_{v}^{*}=\frac{\beta N_{v}\left(R-1-p / \mu_{h}\right)}{R(\beta+M)} .
\end{gathered}
$$

$\beta, M$, and $R$ are given by $\beta=C_{h v} / \mu_{v}, M=\left(\mu_{h}+\gamma_{h}\right) / \mu_{\mathrm{h}}$, and $R=C_{v h} C_{h v}$ $N_{v} /\left(\mu_{v}\left(\mu_{h}+\gamma_{h}\right) N_{h}\right)$. Analysis of the Jacobian at $E_{1}$ and $E_{2}$ shows that $E_{1}$ is globally asymptotically stable if $R \leq 1+p / \mu$ and $E_{2}$ is locally stable if $R>1+p / \mu$.

To develop a deeper understanding of the model we conduct several simulations. Note that all simulations in this study were conducted using Wolfram Mathematica [4].

Our first simulation is based on the variation of vaccination levels of a whole population. We numerically demonstrate the change in outbreak behavior using four levels of total population vaccinated in Figure 2. In Figures 2 and 3, $S_{h}$ is in black, $I_{h}$ is in gray, and $I_{v}$ is dashed.

From Figure 2, we see that if $20 \%$ of the population is vaccinated, the outbreak of the epidemic decreases the number of infected hosts during the outbreak by three times. If half of the population is vaccinated, there is almost no outbreak and if $90 \%$ of the population is vaccinated there is no outbreak. The second scenario is based on the assumption that for different environment temperatures the activity level of mosquitoes differs [5].

For this model, our final simulation is based on the hypothetical size
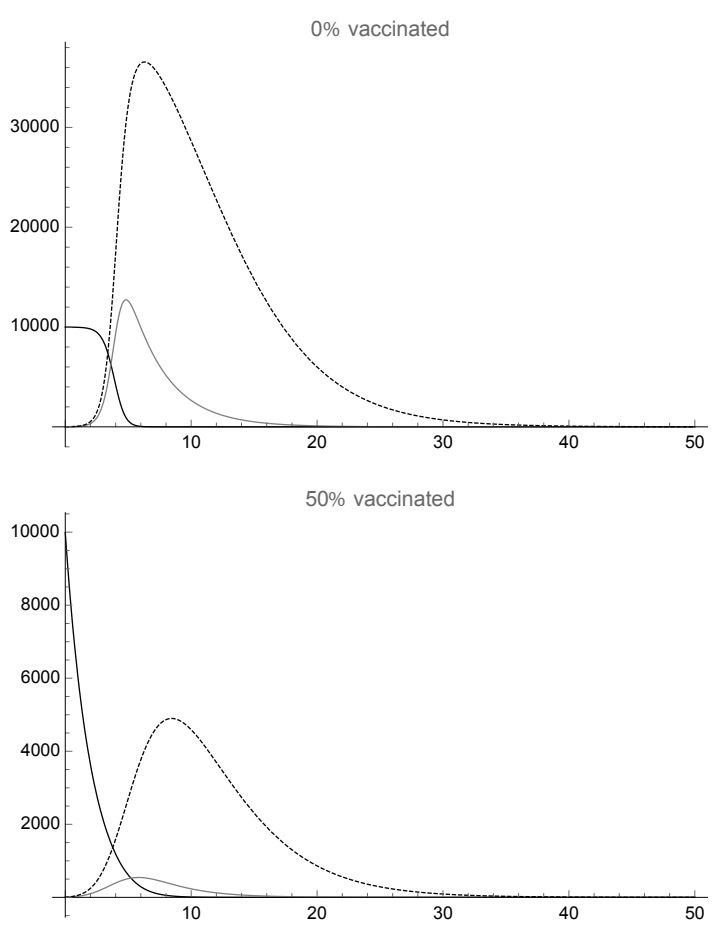

Figure 2: Numerical simulations of the model by Derouich et al. [3] for the different levels of population vaccinated $(0 / 20 / 50 / 90 \%$ of a total population vaccinated). ( $S_{h}$ is in black, $I_{h}$ is in gray, and $I_{v}$ is dashed). of mosquito population and its influence on the size of the outbreak in human population. Figure 3 indicates that if the bite rate of mosquitoes increases, the number of infected mosquitoes increases drastically. Consequently, the size of the outbreak in the human population increases as well. This scenario is important because of global warming and the permanent average temperature on the Earth. Generally, it is thought that warmer weather will cause vectors such as mosquitoes to increase in population size.

The last simulation illustrates the importance of different control measures of mosquito population. In Figure 4, we see that a considerable decrease of mosquito population can almost prevent an outbreak of dengue in the human population.

According to these scenarios it is difficult to identify which parameter affects the severity of an outbreak the most. However, the number of mosquitoes and the vaccination level of the susceptible population appear to be of high importance. Despite the fact that vaccination campaigns can be easily implemented, they are effective only if just one strain of the virus is present in the environment. Otherwise, the vaccination program is a waste of resources. Therefore, the best way to decrease the severity of the outbreak is to reduce the number of mosquitoes.

\section{Feng model of dengue Fever}

Next, we study the model developed by Feng at al. [6]. For the Feng model, the host has size

$$
N=S+I_{1}+I_{2}+Y_{1}+Y_{2}+R
$$

where $S$ represents the number of susceptibles, $I_{i}$ represents the number with primary infection by strain $i, Y_{j}$ represents the number with secondary infection by strain $j$, and $R$ represents the recovered population. For the vector (mosquitoes), $T=M+V_{1}+V_{2}$, where $M$
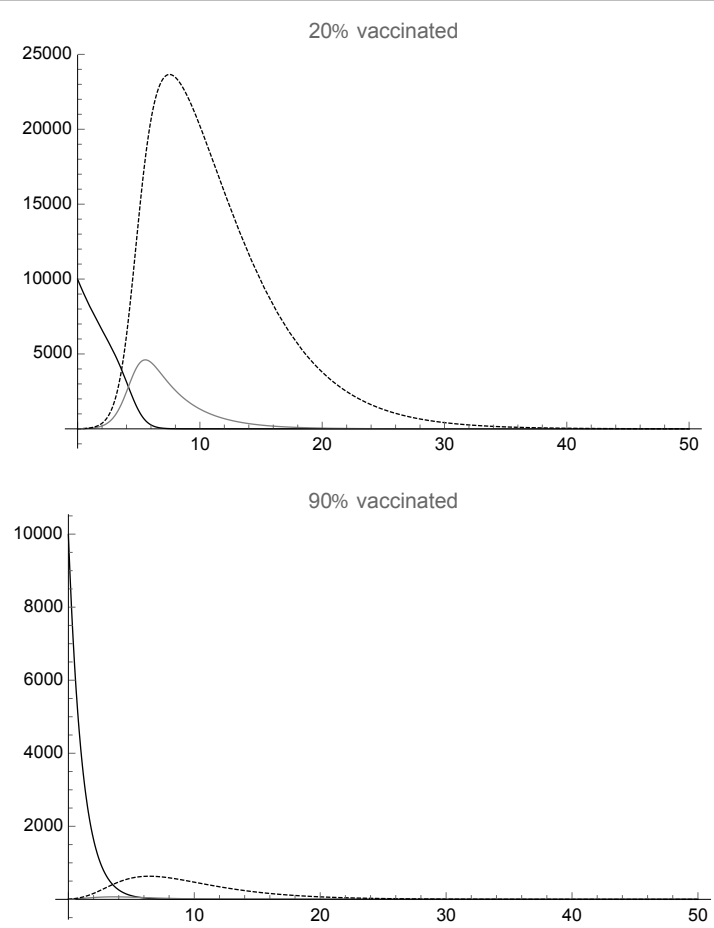

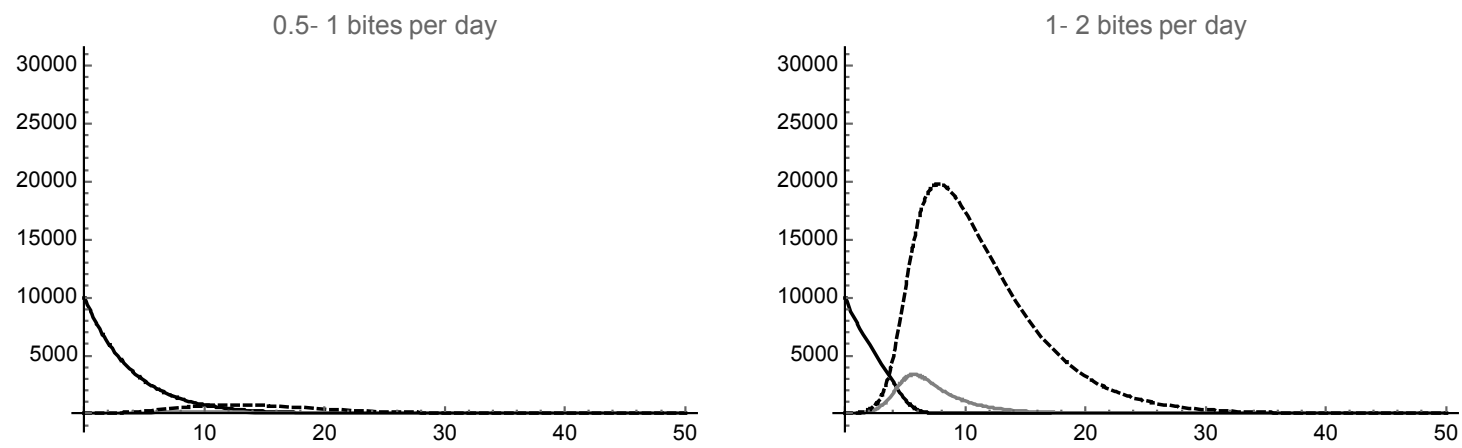

1.5- 2.25 bites per day
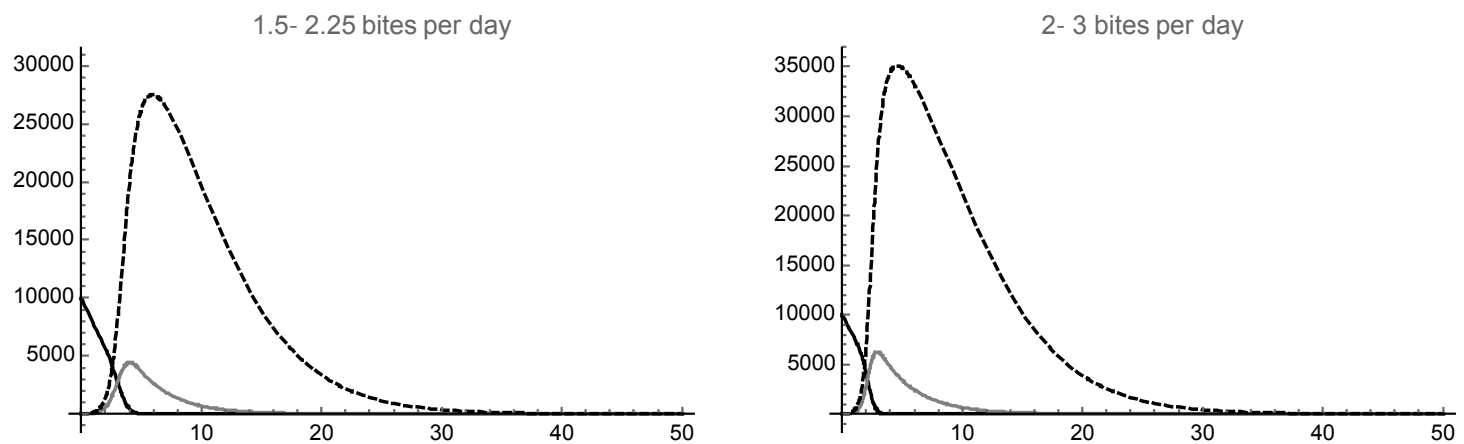

Figure 3: Numerical simulations of the model by Derouich et al. [3], for different levels of mosquito activity (0.5-1/1-2/1.5-2.25/2-3) bites per susceptible/ infectious mosquito per day). ( $S_{h}$ is in black, $I_{h}$ is in gray, and $I_{v}$ is dashed).
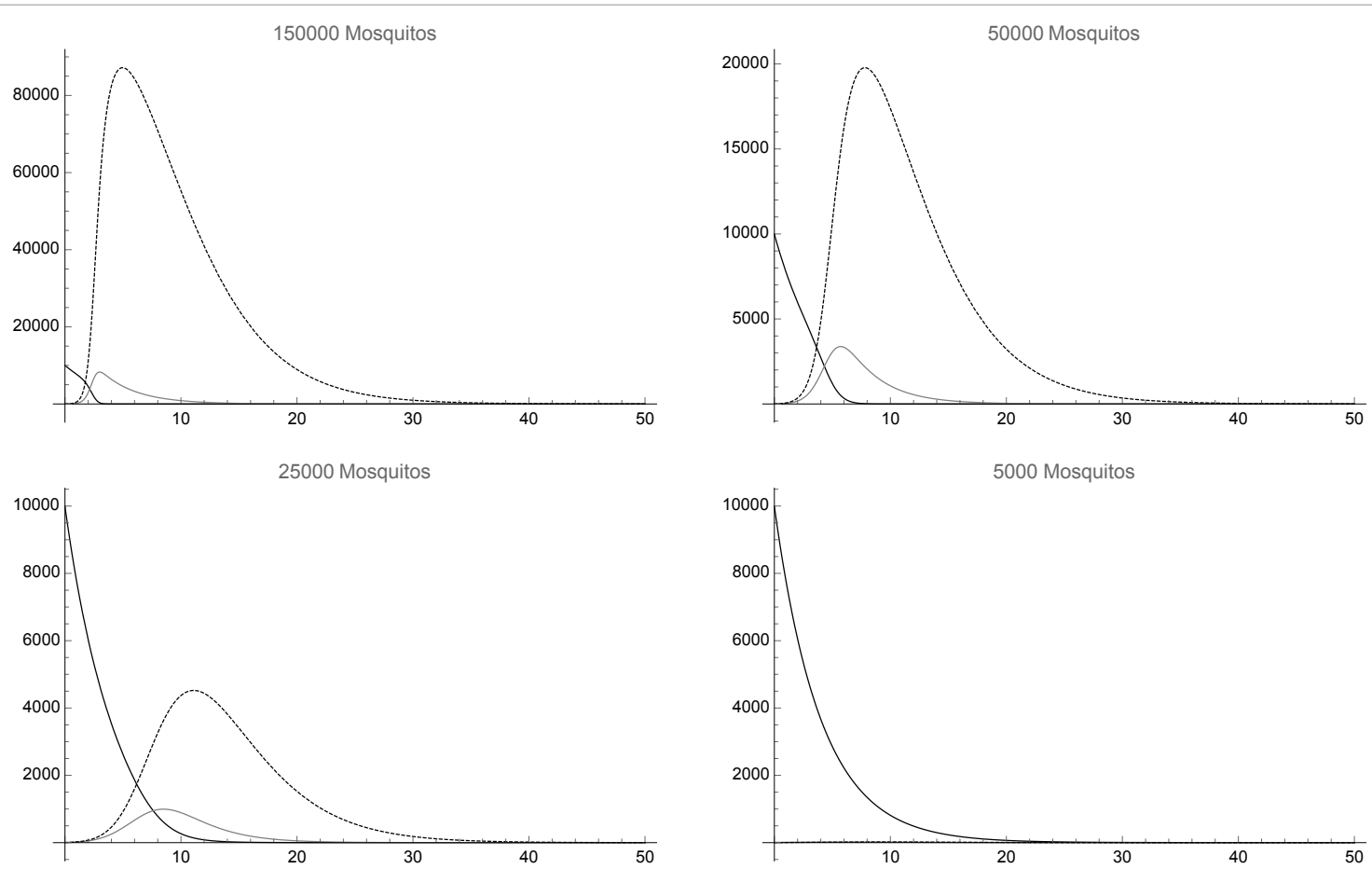

Figure 4: Numerical simulations of the model by Derouich et al. [3] for different levels of mosquito population (150000/50000/25000/5000). ( $S_{h}$ is in black, $I_{h}$ is in gray, and $I_{v}$ is dashed).

represents the number not infected and $V_{i}$ represents the number infected by strain $i$. The model assumes that the vector can only be infected by a single strain of the virus. The remaining parameter values are defined in Table 2 . The model constructed by Feng at al. [6], is 


$$
\begin{aligned}
& \frac{d S}{d t}=h-\left(B_{1}+B_{2}\right) S-u S \\
& \left.\frac{d I_{1}}{d t}=B_{1} S-\sigma_{2} B_{2} I_{1}\right)-u I_{1} \\
& \left.\frac{d I_{2}}{d t}=B_{2} S-\sigma_{1} B_{1} I_{2}\right)-u I_{2} \\
& \frac{d Y_{1}}{d t}=\sigma_{1} B_{1} I_{2}-\left(e_{1}+u+r\right) Y_{1} \\
& \frac{d Y_{2}}{d t}=\sigma_{2} B_{2} I_{1}-\left(e_{2}+u+r\right) Y_{2} \\
& \frac{d R}{d t}=r\left(Y_{1}+Y_{2}\right)-u R
\end{aligned}
$$

and

$$
\begin{aligned}
& \frac{d M}{d t}=q-\left(A_{1}+A_{2}\right) M-\delta M \\
& \frac{d V_{1}}{d t}=A_{1} M-\delta V_{1} \\
& \frac{d V_{2}}{d t}=A_{2} M-\delta V_{2}
\end{aligned}
$$

In (4) and (5), primary infections in humans are produced at rate

$$
B i=\frac{\beta_{i} V_{i}}{C+\omega_{h} N}
$$

by the vector infected with strain $i$. Similarly, infections in vectors (mosquitos) are produced at rate

$$
A_{i}=\frac{a_{i}\left(I_{i}+Y_{i}\right)}{c+\omega_{v} N}
$$

The main result of Feng at al. [6], is that the system (4) has two equilibrium points, $E_{2}{ }^{*}=\left(S_{2}{ }^{*}, 0, I_{1}{ }^{*}, 0,0,0,0, V_{1}^{*}\right)$ and $E_{2}{ }^{*}=\left(S_{2}{ }^{*}, 0, I_{1}^{*}, 0,0,0,0, V_{1}^{*}\right)$. To obtain the precise result on the existence and stability properties of these equilibrium points it was assumed that dengue does not produce significant mortality. So, the dimension of the model was reduced by one. Finally, two equilibrium values were considered: $E_{2}{ }^{*}=\left(0,0, V_{2}{ }^{*}, I_{2}{ }^{*}, 0,0,0,\right)$ and $E_{2}{ }^{*}=\left(0,0, V_{2}^{*}, I_{2}{ }^{*}, 0,0,0,\right)$ (Table 2).
Where $V_{i}^{*}=\frac{\mu \delta\left(R_{i}-1\right)}{b_{i}\left(\delta+a_{i} N\right)}$ and $I_{i}^{*}=\frac{\mu \delta\left(R_{i}-1\right)}{a_{i}\left(b_{1} T+u\right)}, i=1,2$

and $a_{i}=\frac{a_{i}}{c+\omega N}$ and $b_{i}=\frac{\beta_{i}}{c+\omega N}, i=1,2$

For each of the equilibrium points the parameters are defined as

$$
\begin{aligned}
& \sigma_{1}^{*}=\max \left\{0,\left(\frac{R_{2}}{R_{1}}-1\right) \frac{\delta+a_{1} N}{\delta\left(R_{1}-1\right)}\right\}, \\
& f\left(\sigma_{1}\right)=\left(\sigma_{2}\right)^{*}=\left(\frac{\delta(u+r)}{a_{2} b_{2} I_{1}^{*}\left(T-V_{1}^{*}\right)}\right)\left(1-\frac{u R_{2}}{R_{1}\left(u+\sigma_{1} b_{1} I_{1}^{*}\right)}\right)
\end{aligned}
$$

For $E_{1}^{*}$ and

$$
\begin{aligned}
& \sigma_{2}^{+}=\max \left\{0,\left(\frac{R_{2}}{R_{1}}-1\right) \frac{\delta+a_{2} N}{\delta\left(R_{2}-1\right)}\right\}, \\
& g\left(\sigma_{2}\right)=\left(\sigma_{1}\right)^{+}=\left(\frac{\delta(u+r)}{a_{1} b_{1} I_{2}^{*}\left(T-V_{2}^{*}\right)}\right)\left(1-\frac{u R_{1}}{R_{2}\left(u+\sigma_{2} b_{2} I_{2}^{*}\right)}\right)
\end{aligned}
$$

For $E_{1}^{*}$ respectively

Analysis of $E_{1}^{*}$ and $E_{2}{ }^{*}$ shows that:

- $E_{1}^{*}$ is locally asymptotically stable if $\sigma_{2}<f\left(\sigma_{1}\right)$ for every $\sigma_{1}>1$, and unstable if $\sigma_{2}>f\left(\sigma_{1}\right)$

- $E_{2}{ }^{*}$ is locally asymptotically stable if $\sigma_{2}<g-1\left(\sigma_{1}\right)$ for every $\sigma_{1}>1$, and unstable if $\sigma_{2}>g-1\left(\sigma_{1}\right)$

- $E_{1}{ }^{*}$ and $E_{2}{ }^{*}$ are locally asymptomatically stable if $g-1\left(\sigma_{1}\right)<\sigma_{2}<f$ $\left(\sigma_{1}\right)$

For the model three different scenarios were considered and simulations conducted:

(1) Different numbers of mosquito population.

(2) Different mosquito recruitment rate.

(3) Different mosquito activity levels.

The first scenario (Figure 5) demonstrates that a considerable decrease in a mosquito population can significantly decrease the size of the outbreak. From the figure we see that the dependence is almost linear. If we decrease the number of mosquitoes by two times, we obtain nearly a $50 \%$ decrease of infected population. Another interesting observation is that the day when the peak of the outbreak is reached remains the same and does not depend on the number of mosquitoes. In Figure 5, the first graphic represents $S$ in black and $R$ in gray, the second graphic represents $I_{1}$ and $I_{2}$ in black and dashed black and $Y_{1}$ and $Y_{2}$ in grey and dashed grey respectively. The third graphic represents $M$ in black and $V_{1}$ and $V_{2}$ in gray.

\section{Parameter}

Host recruitment rate

Host life expectancy

Mean length of infectious period in host

Vector per capita infection rate (biting rate $\times$ vector infection probability)

Host per capita infection rate (biting rate $\times$ host infection probability)

Vector recruitment rate

Vector life expectancy

Rescaling parameter ( $\alpha i / c$ and $\beta i / c$ infection rates when $N$ small)

Saturation parameter ( $\alpha i / \omega i$ and $\beta i / \omega i$ give maximum infection rates)

Disease-induced per-capita death rate

Susceptibility to strain $i$

\begin{tabular}{|c|c|}
\hline Notation & Base Value \\
\hline$h$ & variable \\
$u^{-1}$ & 70 years \\
$r^{-1}$ & 14 days \\
$\alpha_{i}$ & $(0,0.05)$ \\
$\beta_{i}$ & $(0,0.05)$ \\
$q$ & variable \\
$\delta^{-1}$ & 14 days \\
$c$ & 1 \\
$\omega_{i}$ & 0.5 \\
$e_{i}$ & variable \\
$\sigma_{i}$ & $(0,5)$ \\
\hline
\end{tabular}

Table 2: Parameter values used following the same choices as in Fang and Velascol [6]. 
The second scenario (Figure 6) demonstrates that the mosquito recruitment rate has almost no impact on the outbreak. However, the mosquito recruitment rate can considerably shift the susceptibleinfected distribution among vectors.

The third scenario (Figure 7) describes the outbreak given different mosquito activity levels. The infection rate represents the probability of getting infected by infected host or infected vector after the bite. So, the more active the mosquitoes become; the rate at which the number of hosts and vectors are getting infected increases. We see that even a slight increase of infection rate can significantly affect the form of the outbreak.

After varying several parameters of the current model we see that both vector population and vector activity level affect the severity of the outbreak. However, even though the mosquito activity level appears to have a higher impact on the outbreak, it seems difficult to control the outbreak. The easier way to control the outbreak is to implement public policies to reduce the size of the mosquito population. Examples of such policies include: destroying sites where larvae develop or using strategies to prevent larvae development when water-filled containers are present. At the same time, simpler approaches such as household screening, air- conditioning and other methods to isolate living areas from mosquitoes are also effective in preventing dengue [2] (Figure 5).

\section{Syafruddin and Noorani Models of dengue Fever}

The third and fourth models of dengue fever studied were developed by Syafruddin and Noorani $[7,8]$ respectively. The parameter values they used in both models are the same and are defined in Table 3 (Figure 6).
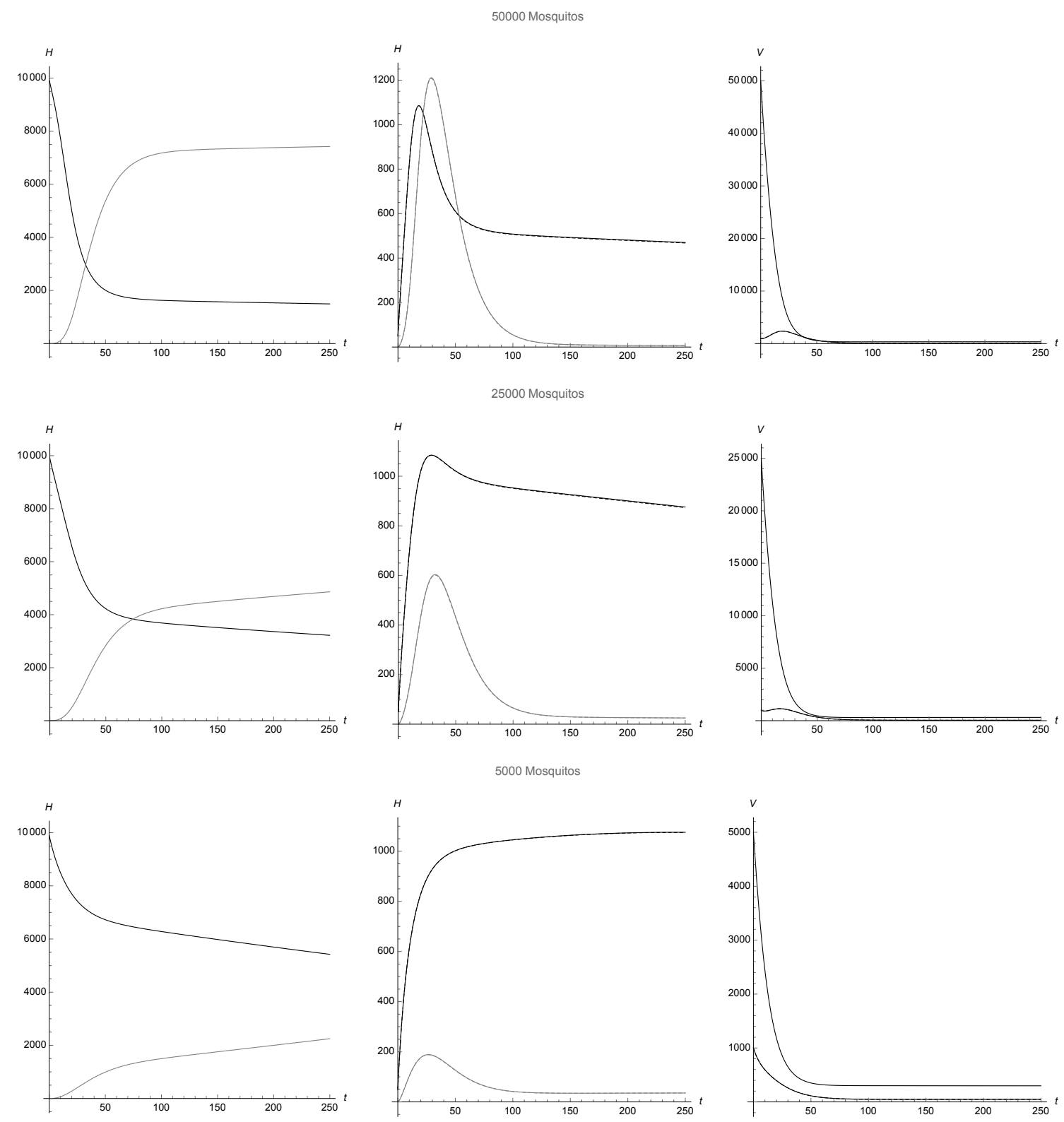

Figure 5: Numerical simulations of the model by Feng et al. [6] for different levels of mosquito population (50000/25000/5000). In the first column, $S$ is black, $R$ is gray; in the second column, $I_{1}$ is black, $I_{2}$ is dashed black, $Y_{1}$ is gray, $Y_{2}$ is dashed gray; in the third column, $M$ is black, $V_{1}$ is gray, $V_{2}$ is dashed gray. 


\begin{tabular}{|c|c|c|}
\hline Parameter & Notation & Base Value \\
\hline $\begin{array}{l}\text { Human Population } \\
\text { Human Birth Rate } \\
\text { Humans Exposed to Virus } \\
\text { Rate Humans Exposed to Virus Proportionality Constant } \\
\text { Death Rate of Humans } \\
\text { Recovery Rate of Infected Humans } \\
\text { Susceptible Human Population } \\
\text { Infected Human Population } \\
\text { Death Rate of Infected Human Population } \\
\text { Recovered Human Population } \\
\text { Vector Population } \\
\text { Percentage of Vector Population Infected } \\
\text { Susceptible Vector Population } \\
\text { Death Rate of Vectors Infected } \\
\text { Vector Population Death Rate } \\
\text { of Infected Vectors } \\
\text { Average Number of Bites per Infected Mosquito } \\
\text { Probability of Uninfected Vector being Infected by Human } \\
\text { Probability of Uninfected Human being Infected by Vector }\end{array}$ & $\begin{array}{c}N_{h} \\
\mu_{h} \\
E_{h} \\
\varphi_{h} \\
\mu_{h} \\
V_{h} \\
S_{h} \\
I_{h} \\
a_{h} \\
R_{h} \\
N_{v} \\
p \\
S_{v} \\
\mu_{v} \\
I_{v} \\
V_{v} \\
b \\
\beta h\left(b \beta_{h}\right) \\
\beta v\left(V=b \beta_{v}\right)\end{array}$ & $\begin{array}{c}\text { variable } \\
0.0045 \\
\text { variable } \\
0.16667 \\
\text { variable } \\
0.328833 \\
\text { variable } \\
\text { variable } \\
0.0000002 \\
\text { variable } \\
\text { variable } \\
0.09 \\
\text { variable } \\
0.02941 \\
\text { variable } \\
\text { variable } \\
\text { variable } \\
0.75 \\
(0.375)\end{array}$ \\
\hline
\end{tabular}

Table 3: Parameter values used by Syafruddin and Noorani $[7,8]$.

50 Mosquito Recruitment Rate
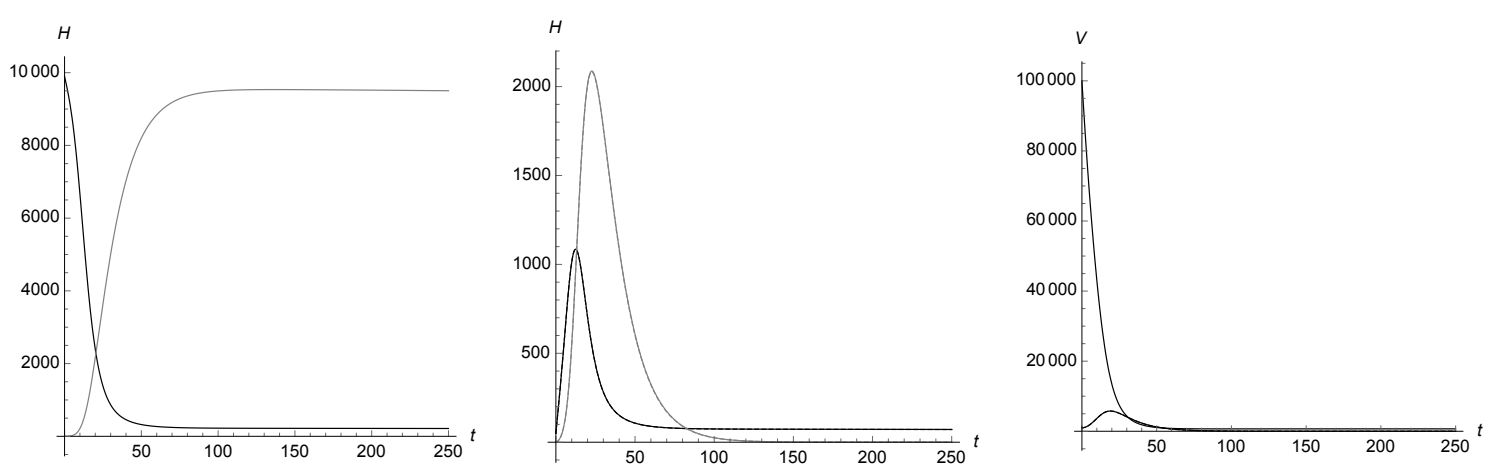

750 Mosquito Recruitment Rate
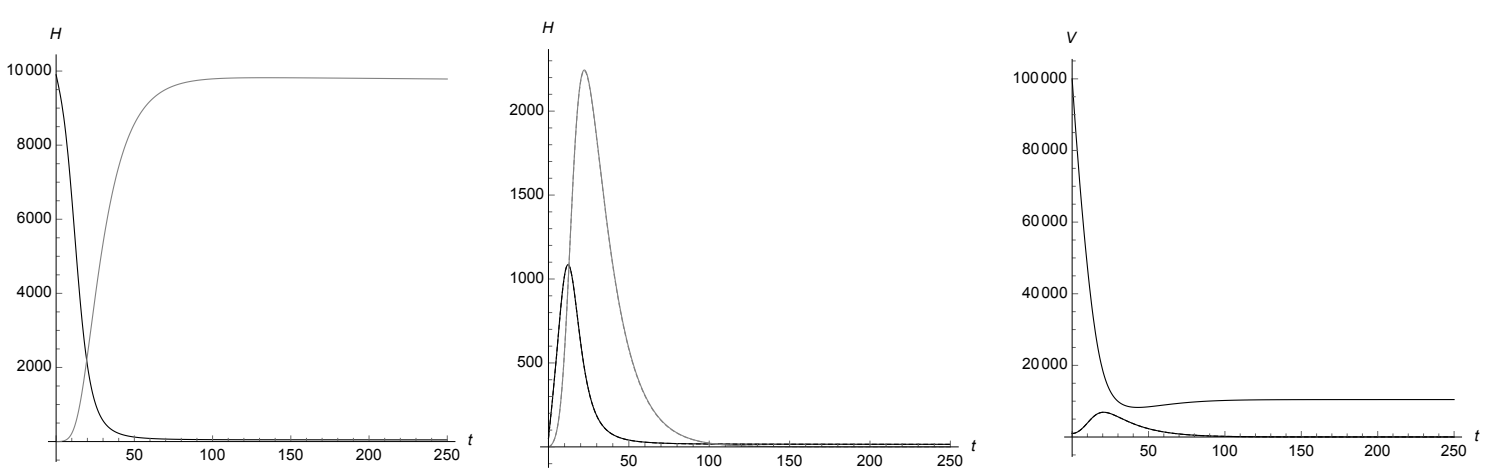

Figure 6: Numerical simulations of the model by Feng et al. [6] for different levels of mosquito recruitment rate (50/750). In the first column, $S$ is black, $R$ is gray; in the second column, $I_{1}$ is black, $I_{2}$ is dashed black, $Y_{1}$ is gray, $Y_{2}$ is dashed gray; in the third column, $M$ is black, $V_{1}$ is gray, $V_{2}$ is dashed gray.

\section{The first Syafruddin and Noorani model}

The susceptible-infected recovered $(S-I-R)$ model used by Syafruddin and Noorani in ref. [8] simplifies to

$$
\begin{aligned}
& \frac{d x}{d t}=\mu_{h}(1-x)-a x z \\
& \frac{d y}{d t}=a x z-\beta y \\
& \frac{d z}{d t}=\gamma(1-z) y-\delta z .
\end{aligned}
$$

In system (6), and $\delta=\mu_{v}$. The parameter values are described in Table 3. The probability of a susceptible human being infected with dengue is $\frac{\beta_{h} b I_{v}}{N_{h}}$.

The main result of the first Syafruddin and Noorani model, [7] is that system (6) has two equilibrium points $E_{1}=(1,0,0)$ and $E_{2}=\left(x_{0}, y_{0}\right.$, $z_{0}$ ) with the values: 


$$
x_{0}=\frac{\mu_{h} \gamma+\beta \delta}{\gamma\left(\mu_{h}+\alpha\right)}, y_{0}=\frac{\mu_{H}(\gamma \alpha+\beta \delta)}{\beta \gamma\left(\mu_{H}+\alpha\right)}, \text { and } z_{0}=\frac{\mu_{H}(\gamma \alpha+\beta \delta)}{\alpha\left(\gamma \mu_{H}+\beta \delta\right)}
$$

Analysis of those equilibrium points for the South Sulawesi outbreak shows that $E_{1}$ is globally asymptotically stable point and $E_{2}$ is asymptotically stable point [7] (Figure 7).

To illustrate the behavior of this model several simulations were performed. In the first simulation we assumed that the proportions of susceptible and infected population can vary initially (Figure 8 and Table 3). As shown in Figure 8, this scenario illustrates that the more people initially infected, the faster the remaining susceptible population will decrease.
The second scenario describes the situation with different activity levels of mosquitoes. Figure 9 illustrates different situations depending on the probability of the mosquito to infect a human during a bite. We see that if the probability of being bitten by an infected mosquito is relatively small, there will not be any outbreak. However, if the probability of being bitten by an infected mosquito becomes high, the outbreak can be very severe with a high peak.

The last scenario describes the situation when we have different proportions of initially infected mosquitoes. Interestingly, Figure 10 indicates that if initially not all mosquitoes are infected, we obtain a shift in the peak of the outbreak along with the decrease of the severity of the outbreak. At the same time, the percentage of infected mosquitoes will
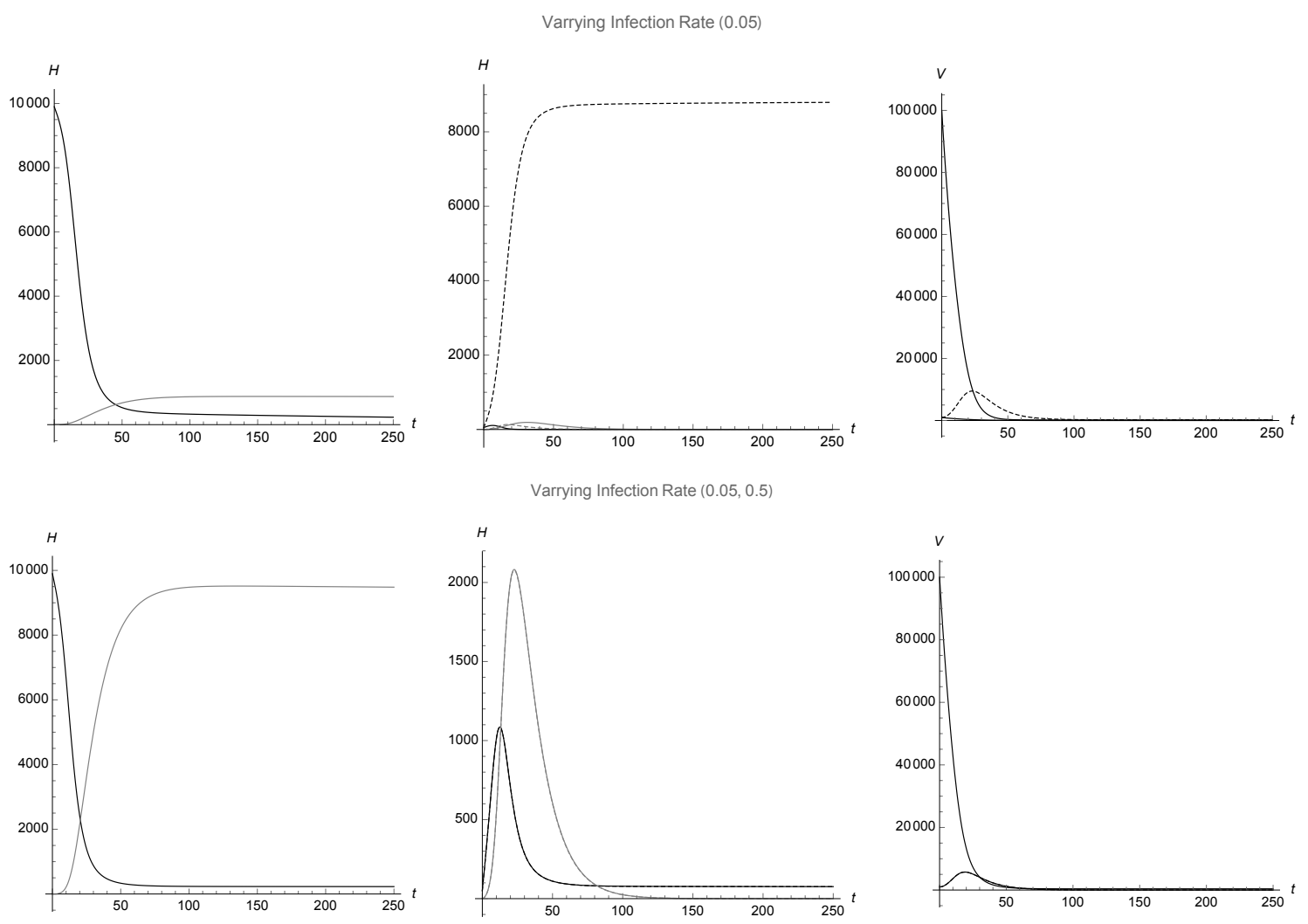

Varrying Infection Rate $(0.1,0.5)$
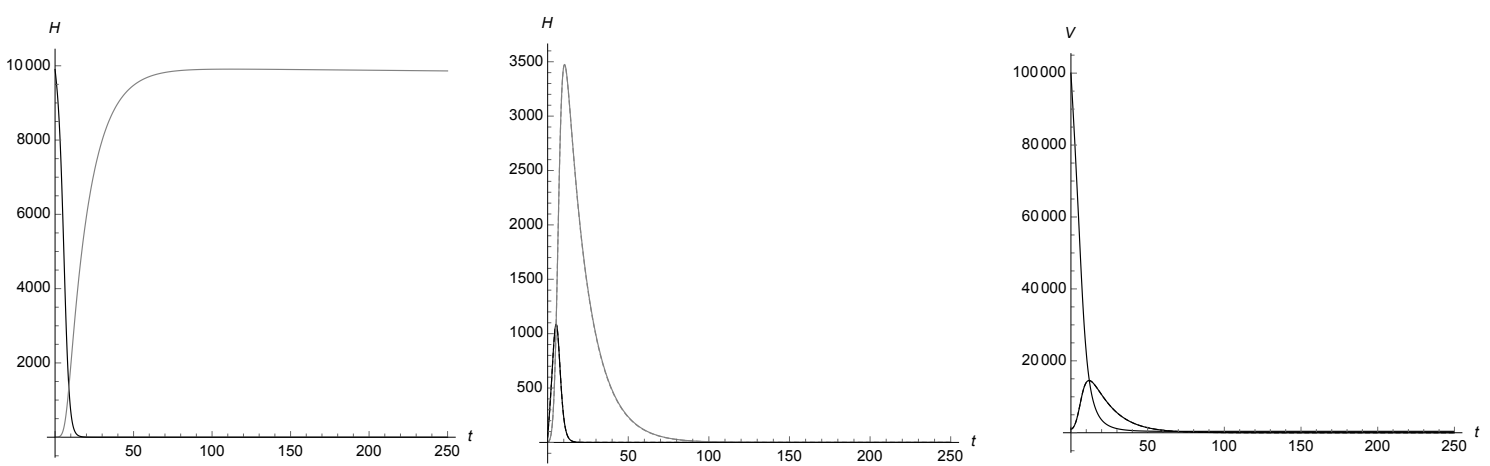

Figure 7: Numerical simulations of the model by Feng et al. [6] for different levels of infection rate $(0.05,0.05 / 0.05,0.5 / 0.1,0.5)$. In the first column, $S$ is black, $R$ is gray; in the second column, $I_{1}$ is black, $I_{2}$ is dashed black, $Y_{1}$ is gray, $Y_{2}$ is dashed gray; in the third column, $M$ is black, $V_{1}$ is gray, $V_{2}$ is dashed gray. 


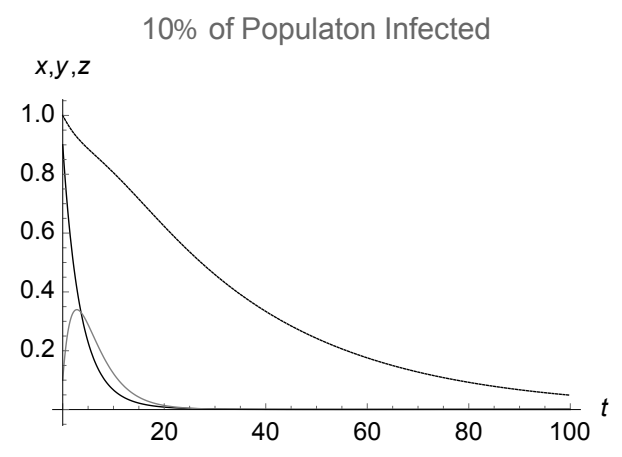

$50 \%$ of Populaton Infected

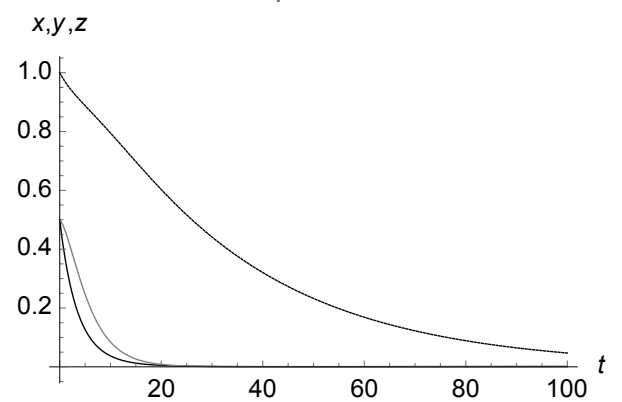

$30 \%$ of Populaton Infected

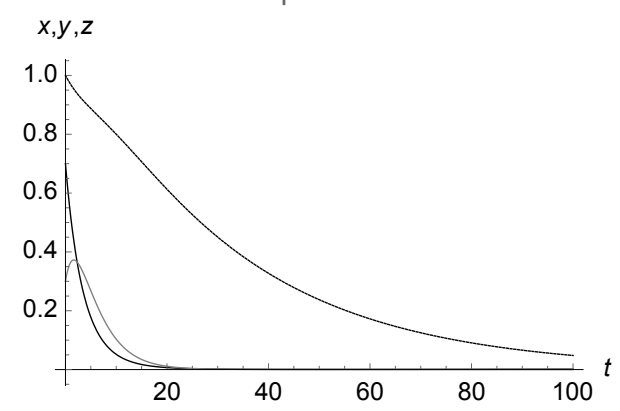

$80 \%$ of Populaton Infected

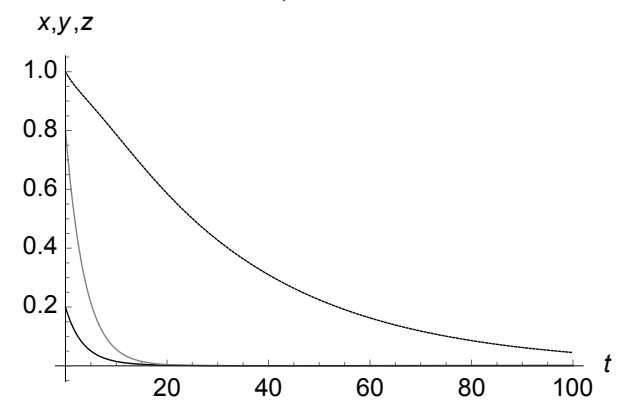

Figure 8: Numerical simulations of the model by Syafruddin et al. [8] for different proportions of susceptible and infected population (0.9-0.1/0.7-0.3/0.5-0.5/0.2-0.8). $x$ is black, $y$ is gray, and $z$ is dashed black.

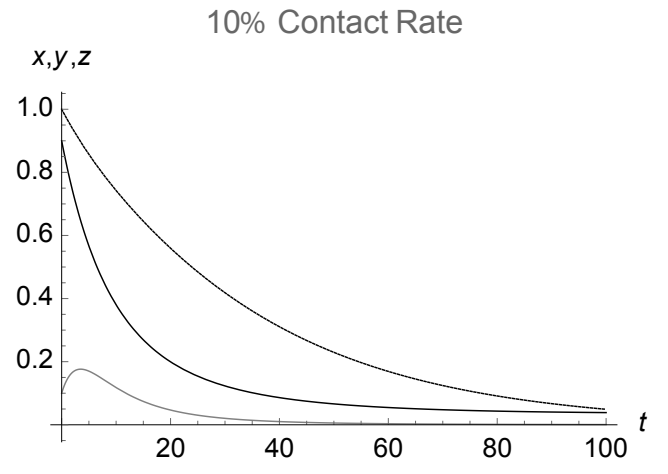

$35 \%$ Contact Rate

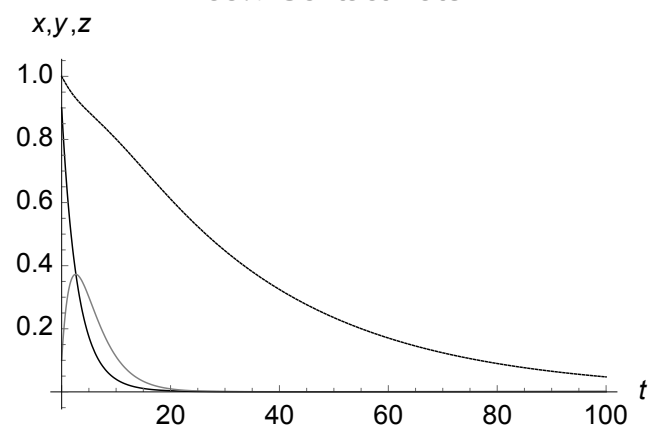

$15 \%$ Contact Rate

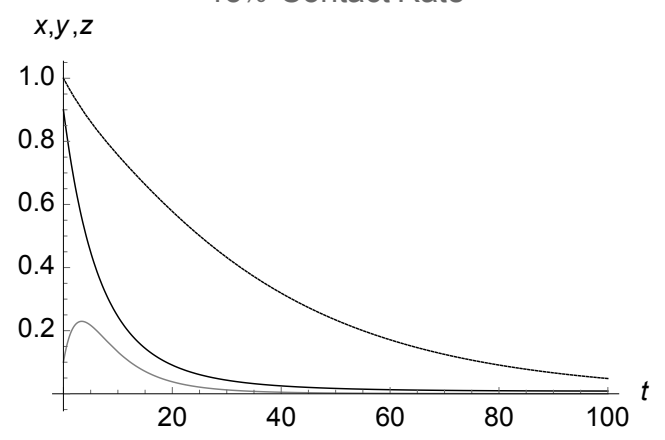

70\% Contact Rate

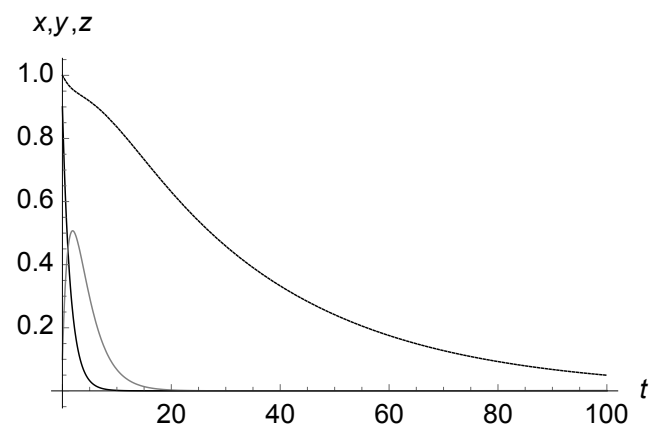

Figure 9: Numerical simulations of the model by Syafruddin et al. [8] for different probabilities of humans being bitten by a mosquito and being infected by Dengue $(0.1 / 0.15 / 0.35 / 0.7) . x$ is black, $y$ is gray, and $z$ is dashed black. 
$100 \%$ Infected Mosquitos

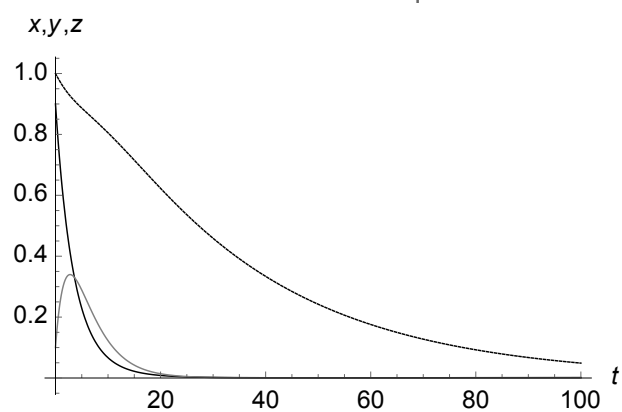

$25 \%$ Infected Mosquitos

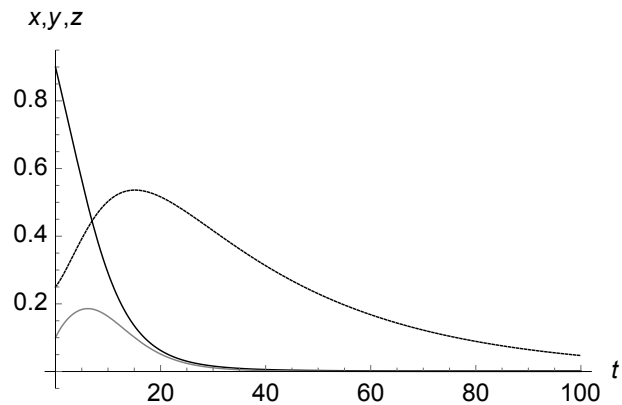

$60 \%$ Infected Mosquitos

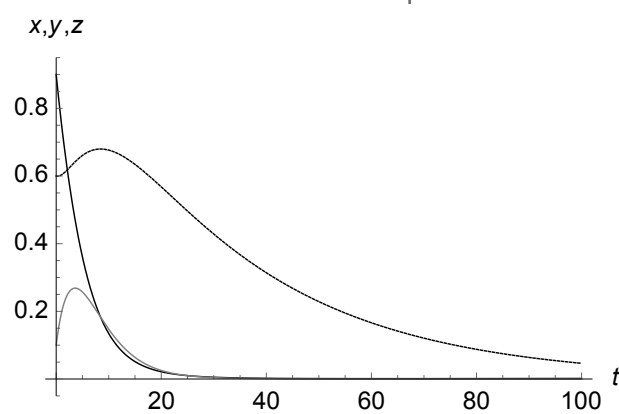

$0.01 \%$ Infected Mosquitos

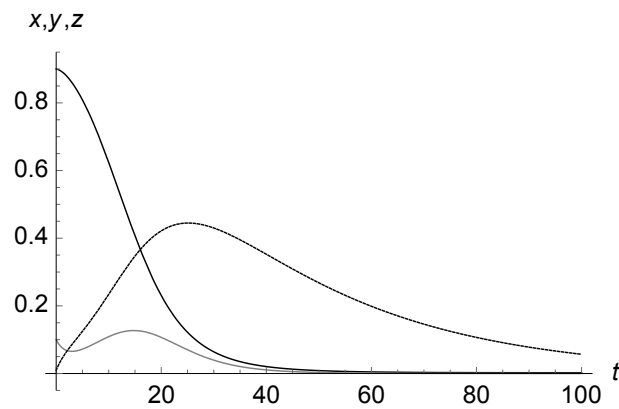

Figure 10: Numerical simulations of the model by Syafruddin et al. [8] for different proportions of initially infected mosquitoes (1/0.6/0.25/0.01). ( $x$ is black, $y$ is gray, and $z$ is dashed black.

grow and reach a maximum. However, a situation in which nearly all mosquitoes would be infected is virtually impossible.

\section{The second Syafruddin and Noorani model}

Using $\quad x=\frac{S_{h}}{N_{h}}, u=\frac{E_{h}}{N_{h}}, y=\frac{I_{h}}{N_{h}}, \omega=\frac{E_{v}}{N_{v}}$, and $z=\frac{I_{v}}{N_{V}}, \quad$ the susceptible-exposed-infected-recovered $(S-E-I-R)$ model used by Syafruddin and Noorani in ref. [8] simplifies to

$$
\begin{aligned}
& \frac{d x}{d t}=\mu_{h}(1-x)-(\alpha z+p) x \\
& \frac{d u}{d t}=(\alpha z+p) x-\left(\mu_{h}+\phi_{h}\right) u \\
& \frac{d y}{d t}=\phi_{h} u-\left(\mu_{h}+\gamma_{h}+\alpha_{h}\right) y \\
& \frac{d w}{d t}=\gamma_{v}(1-z-w) y-\left(\mu_{v}+\delta_{v}\right) w \\
& \frac{d z}{d t}=\delta w-\mu_{v} z,
\end{aligned}
$$

Where, $\alpha=\frac{\beta_{h} b N_{v}}{N_{h}}$

Refer to Table 3 for the meanings of the parameter values. The main result of the second Syafruddin and Noorani model, [8], is that system (7) has one equilibrium point $E_{1}$. This time, equilibrium points were numerically calculated for the Selangor (Malaysia) outbreak data and $E_{1}$ is asymptotically stable.
In comparison to the previous models, the scaled Syafruddin and Noorani models are convenient because $x, u, y, w$, and $z$ represent population percents rather than specific numbers. This makes it easier to compare the effects of the virus on the mosquito and human populations.

\section{Nuraini Model of Dengue Fever}

For, $i, j=1,2, i \neq j$ the normalized dengue model developed by Nuraini et al. [9], takes the form

$$
\begin{aligned}
& \frac{d S}{d t}=\left(1-\mu_{h}\right) S-\left(B_{1} V_{1}+B_{2} V_{2}\right) S \\
& \frac{d I_{i}}{d t}=B_{i} V_{i}-(\gamma+\mu h) I_{i} \\
& \frac{d R i}{d t}=\gamma I_{i}-\sigma B_{j} V_{j} R_{i}-\mu_{h} R_{i} \\
& \frac{d D}{d t}=q\left(\sigma_{2} B_{2} V_{2} R_{1}+\sigma_{1} B_{1} V_{1} R_{2}\right)-\left(\mu_{h}+\gamma\right) D \\
& \frac{d Y_{i}}{d t}=(1-q) \sigma_{i} B_{i} V_{i} R_{j}-\left(\gamma+\mu_{h}\right) Y_{i} \\
& \frac{d V_{i}}{d t}=A_{i}\left(I_{i}+Y_{i}\right)\left(1-V_{1}-V_{2}\right)-\mu_{v} V_{i}
\end{aligned}
$$

In system (8), for the host, $\mathrm{S}+\mathrm{I}_{1}+\mathrm{I}_{2}+\mathrm{Y}_{1}+\mathrm{Y}_{2}+\mathrm{R}+\mathrm{D}=1$. $S$ represents the percent of the population susceptible, $I_{i}$ the percent infected with strain $i, R_{i}$ represents the percent immune to strain $i, Y_{i}$ the percent of the population immune to strain $j(j=2,1)$ but are infected with strain $i(i=1,2), R$ the percent immune to both strains, and $D$ the percent for 

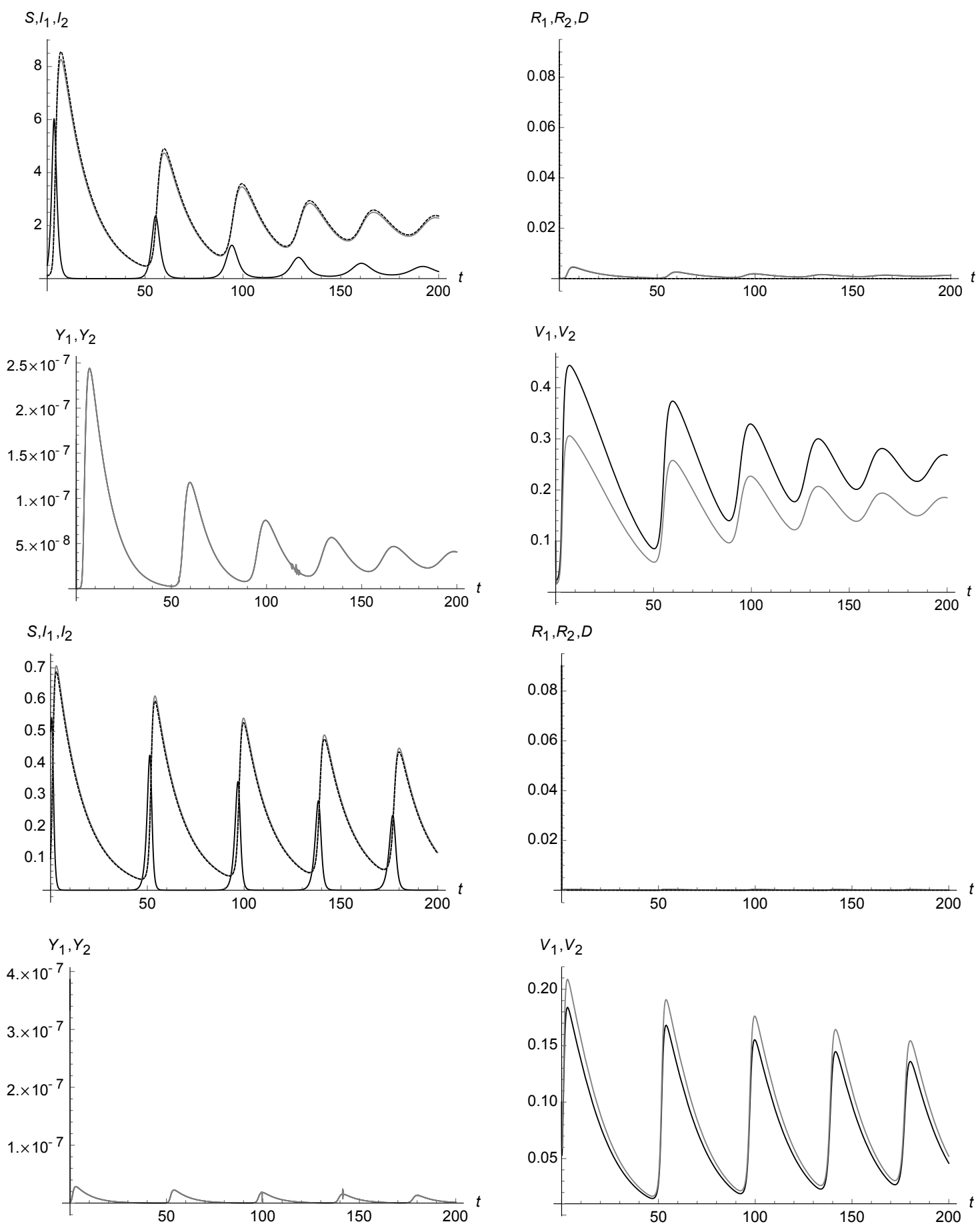

Figure 11: Numerical simulations for the model by Nuraini et al. [9], for different levels of mosquito activity $(3,2 / 7,6)($ These numbers represent the number of susceptible hosts which can be infected by an infected vector and vice versa). ( $S$ is black, $I_{1}$ is gray, $I_{2}$ is dashed black, $R_{1}$ is black, $R_{2}$ is gray, $D$ is dashed black, $Y_{1}$ is black, $Y_{2}$ is gray, $V_{1}$ is black, and $V_{2}$ is gray.

those who are immune to one strain but become infected with the other strain and develop severe symptoms (severe dengue Hemorrhagic fever). For the vector (mosquitoes), $V_{0}+V_{1}+V_{2}=1 . V_{i}$ represents the percent infected with strain $i$. The parameter values are listed in Table 4.

The main result of the Nuraini et al. model [9] is that the system (8) has 4 equilibrium points: $E_{0}=(1,0,0,0,0,0$,
$0, \quad 0, \quad 0, \quad 0), \quad E_{2}=\left(\mathrm{S}_{2}^{*}, 0, \mathrm{I}_{2}{ }^{*}, 0, \mathrm{R}_{2}^{*}, 0,0,0,0, \mathrm{~V}_{2}^{*}\right)$, $E_{2}=\left(\mathrm{S}_{2}{ }^{*}, 0, \mathrm{I}_{2}{ }^{*}, 0, \mathrm{R}_{2}{ }^{*}, 0,0,0,0, \mathrm{~V}_{2}{ }^{*}\right)$, where

$$
\begin{aligned}
& S_{i}^{*}=\frac{\mu_{h} T_{i}+B_{i}}{T_{i}\left(\mu_{h}+B_{i}\right)}, I_{i}^{*}=\frac{\mu_{h} B_{i}\left(T_{i}-1\right)}{\left(\mu_{h}+\gamma\right)\left(\mu_{h}+B_{i}\right) T i}, \\
& R_{i}^{*}=\frac{\gamma I_{i}^{*}}{\mu_{h}\left(\mu_{h}+\gamma\right)}, V_{i}^{*}=\frac{\mu_{h}\left(T_{i}-1\right)}{\mu_{h} T_{i}+B_{i}}, i=1,2
\end{aligned}
$$




\section{Parameter}

Notation

$\mu h^{-1}$
$\mu v^{-1}$
$\gamma^{-1}$
$A_{i}$
$B_{i}$
$\sigma_{i}$
$q$

Table 4: Parameter values used by Nuraini et al. [9].

and $E_{3}=\left(S^{* *}, I_{i}^{* *}=I^{* *}, R_{i}^{* *}=R^{* *}, Y_{i}^{* *}=Y^{* *}, D^{* *}\right)$, where

$$
\begin{aligned}
& S^{* *}=\frac{\mu_{h}}{\mu_{h}+2 B V^{* *}} I_{i}^{* *}=\frac{B V^{* *} S^{* *}}{\mu_{h}+\gamma} R_{i}^{* *}=\frac{\gamma I^{* *}}{\sigma B V^{* *}+\mu_{h}} \\
& Y_{i}^{* *}=\frac{(1-q) \sigma B V^{* *} R^{*}}{\mu_{h}+\gamma} D^{* *}=\frac{2 q\left(\mu_{h}+\gamma\right) Y^{* *}}{(1-q)\left(\mu_{h}+\gamma\right)} i=1,2
\end{aligned}
$$

For each of those points the following results were obtained:

$E_{0}$ is locally asymptotically stable if and only if $T_{i}<1$.

$E_{1}$ and $E_{2}$, equilibrium points for one serotype are locally asymptotically stable when

$$
T_{i}>1 \text { and } T_{j}<\frac{T_{i}}{1+\frac{\gamma \sigma_{j} B_{i}(1-q)\left(T_{i}-1\right)}{\mu_{h}\left(T_{i}+B_{i}\right)\left(\mu_{h}+\gamma\right)^{2}}}, \quad i, j=1,2, i \neq j
$$

The last equilibrium point $E_{3}$ unlike the previous ones represents the coexistence of two serotypes of viruses. It is locally asymptotically stable if and only if

$$
1<T<\frac{B\left(B \sigma \mu_{v}+2 A \mu_{h}^{2}+\Gamma(2+\sigma)\right)}{2 \mu_{h} \Gamma}+1 \quad \Gamma=\mu_{h} \mu_{v}\left(\mu_{h}+\gamma\right)
$$

where

$$
T_{i}=\frac{A_{i} B_{i}}{\mu_{v}\left(\mu_{h}+\gamma\right), \quad i=1,2}
$$

and is defined as the expected number of cases in individuals of type 1 caused by the infected individual of type 1 in a completely susceptible population.

As for previous models, we also explored the behavior of this model based on several different scenarios. The first type of scenarios describes different biting rates of mosquitoes. As described above, with an increase of atmospheric temperature, mosquitoes become more active and, consequently, the probability to infect an individual increases.

It can be observed that those graphs are different from all previous, which can be explained by the fact that this model describes not only an epidemic outbreak of the disease but the endemic situation of the disease. According to this model, after the end of the outbreak the disease will not vanish, but will remain at a very low level until the required number of susceptible hosts will not reappear in the environment. After that, a new outbreak will take place in the susceptible population.

As we can observe from the top left graphic in Figure 11, each subsequent out- break is smaller than the previous one. This phenomenon is because of the immunity of the percentage of the population that had a disease during a previous outbreak. Also, it can be observed that with the increase of the activity of the vectors (mosquitoes), peaks of the outbreaks become sharper. However, this does not mean that the number of infected hosts grows (Table 4).
Unlike some of the previous models, this model does not take deaths into account. However, when compared to the previous models, this model appears to be the most comprehensive because it attempts to capture only the most relevant parameters. For example, compare the number of values used in system (7) to those used in system (8).

\section{Conclusion}

\section{General conclusions}

This paper reviewed several continuous mathematical models of dengue fever. Five models with different approximations to modelling and different assumptions were considered and for each of them several outbreak scenarios were reviewed.

It was observed that every model is different. The models by Derouich et al. [3] and by Syafruddin et al. [7] are among the simplest one. Both of them are $S-I-R$ continuous dynamical system models of one strain of the virus. The model developed by Feng et al. [6] is a more complicated one. This one is also $S-I-R$ continuous dynamical system model, but of two different strains of dengue.

The most comprehensive model is developed by Nuraini et al. [9]. It not only describes the outbreak with two strains, but also takes into account the separate severe dengue Hemorrhagic Fever state which is not taken into consideration in any of previous models. In addition, this model describes the endemic behavior of the disease, whereas the other models are modelling only epidemic outbreak.

The other interesting model was developed by Syafruddin et al. [8]. This is the only example of $S-E-I-R$ model considered here, which divide the whole human population into four compartments: susceptible, exposed, infected and recovered (removed).

On the next step in the investigation several hypothetic scenarios for each of the outbreaks were conducted to investigate the behavior of the each model and try to answer the question, "which intervention can be the most efficient in terms of decreasing the number of infected population?" Two different types of interventions are available to reach those goals: vaccination and the direct decrease of the mosquito's population. Some models show that vaccination can be useful. However, those models assume only one strain of the virus. If there are more strains in the environment vaccination becomes practically useless since currently available vaccines can only protect from one strain, leaving the whole population completely susceptible to others. Therefore, the only feasible working strategy is to decrease the number of mosquitoes.

At the same time another interesting phenomenon was observed. Since the activity of mosquitoes is based on weather condition, mostly on the temperature, global warming will increase the possibility of being infected and, consequently, the risk of outbreaks.

Finally, there are other problems to consider. One is to develop more models to catch observe important features during the progression of 
an epidemic. For example, the development of an $S-E-I-R$ model of two different strains will be a step forward in this direction. The ultimate goal is to build a model that will describe the outbreak of four different strains at the same time. However, even a small increase in complexity of the initial model drastically increases the difficulty of its validation. Moreover, the amount of real data needed for validation also increases and this data is not easy to obtain.

\section{Computational notes}

The graphics and computations in this paper were carried out using Mathematica [4]. Jim Braselton will send you copies of the notebooks used here if you send a request to him at jbraselton@georgiasouthern.edu.

\section{References}

1. WHO (2015) Dengue and severe dengue. Fact sheet No. 117, Media centre, WHO.

2. Cummings DAT, Lesser J (2013) Infectious disease dynamics. In: Nelson KE, Masters WCS (eds.) Infectious disease epidemiology. Jones and Bartlett Learning, pp. 138-139, 830-831.
3. Derouich M, Boutayeb A, Twizell EH (2003) A model of Dengue fever. Bio Medical Engineering Online 2: 4.

4. Wolfram Research Inc. (2015) Mathematica, Version 10.0, Wolfram Research, Inc., Champaign, Illinois.

5. Trykhlib VI (2013) Seasonal activity of mosquitoes anopheles and their influence on malaria morbidity. Main Military Medical Clinical Centre MMCC, Kiev, Ukraine.

6. Zhilan F, Jorge XVH (1997) Competitive exclusion in a vector-host model for the Dengue fever. J Math Biol 35: 523-544.

7. Syafruddin S, Noorani S (2012) SEIR model for transmission of Dengue fever in Selangor Malaysia. International Journal of Modern Physics: Conference Series 9: 380-389.

8. Syafruddin S, Nooran S (2013) An SIR Model for Spread of Dengue fever disease (simulation for South Sulawesi, Indonesia, and Selangor, Malaysia). World Journal of Modeling and Simulation 9: 96-105.

9. Nuraini N, Soewong E, Sidarto KA (2007) Mathematical model of Dengue disease transmission with severe DHF Compartment. Bulletin of the Malaysian Mathematical Sciences Society 30: 143-157. 